\title{
Multipliers with closed range on commutative semisimple Banach algebras
}

by

\author{
A. ÜLGER (Istanbul)
}

\begin{abstract}
Let $A$ be a commutative semisimple Banach algebra, $\Delta(A)$ its Gelfand spectrum, $T$ a multiplier on $A$ and $\widehat{T}$ its Gelfand transform. We study the following problems. (a) When is $\delta(T)=\inf \{|\widehat{T}(f)|: f \in \Delta(A), \widehat{T}(f) \neq 0\}>0$ ? (b) When is the range $T(A)$ of $T$ closed in $A$ and does it have a bounded approximate identity? (c) How to characterize the idempotent multipliers in terms of subsets of $\Delta(A)$ ?
\end{abstract}

Introduction. Let $A$ be a commutative semisimple Banach algebra, $M(A)$ be its multiplier algebra [La1] and $T$ be a multiplier on $A$. We denote by $\Delta(A)$ the Gelfand spectrum of $A$ and by $\widehat{T}$ the Gelfand transform of $T$; the elements of $\Delta(A)$ are considered as multiplicative functionals on $A$. Let $\Delta(\widehat{T})=\{f \in \Delta(A): \widehat{T}(f) \neq 0\}$ and $\delta(T)=\inf \{|\widehat{T}(f)|: f \in \Delta(\widehat{T})\}$. In this paper our aim is to study the following questions. (a) When is $\delta(T)>0$ ? (b) When is the ideal $T(A)$ closed in $A$ ? (c) When does the ideal $T(A)$ have a BAI (= bounded approximate identity)? (d) How to characterize the idempotent elements of $M(A)$ in terms of subsets of $\Delta(A)$ ?

The main motivation behind these questions is a well known problem of harmonic analysis first studied by I. Glicksberg [G] and later completely solved in $[\mathrm{H}-\mathrm{P}]$ by B. Host and F. Parreau. To recall this problem, let $G$ be a locally compact abelian group equipped with its Haar measure, $L^{1}(G)$ be its group algebra and $M(G)$ be the algebra of regular complex Borel measures on $G$. The algebra $M(G)$ is the multiplier algebra of $L^{1}(G)$. For a measure $\mu$ of the form $\mu=\lambda * \theta$, where $\lambda$ is an invertible element and $\theta$ an idempotent element of $M(G)$, the ideal $L^{1}(G) * \mu$ is obviously closed in $L^{1}(G)$. The problem mentioned above, which was raised by E. Hewitt, was this: For a measure $\mu \in M(A)$, is the ideal $L^{1}(G) * \mu$ closed in $L^{1}(G)$ only when the measure $\mu$ is of the above form? That this is the case is the

2000 Mathematics Subject Classification: Primary 47B48, 46J10, 43A22.

Key words and phrases: multiplier, spectrum, Fourier algebra.

Research supported by a fund of the Turkish Academy of Sciences. 
achievement of [H-P]. Thus the ideal $L^{1}(G) * \mu$ is closed in $L^{1}(G)$ iff $\mu$ is the product of an invertible measure with an idempotent one. One of the main ingredients of the solution is the fact that, when $L^{1}(G) * \mu$ is closed in $L^{1}(G), \delta(\mu)>0 .(\delta(\mu)$ is defined in the same way as $\delta(T)$.) Moreover, factorizability of $\mu$ as $\mu=\lambda * \theta$ is equivalent to the existence of a BAI in the ideal $L^{1}(G) * \mu$ (see $[\mathrm{Z}]$ ). The proof of this result given in [H-P] is highly group-theoretical and does not seem susceptible of generalization. The general or abstract version of this problem presents a certain interest given that it is related (a) with the problem of characterization of the complemented ideals of $A$, (b) with the problem of characterization of the idempotent elements of $M(A)$; and (c) with the Weiner-Pitt phenomenon. The abstract version of this problem has been considered in several papers; see the papers $[\mathrm{A}-\mathrm{L}],[\mathrm{L}-\mathrm{M}]$, Chapter 4 of the book $[\mathrm{L}-\mathrm{N}]$ and references there.

In the present paper we also study the same kind of questions in an abstract setting with applications to the Fourier algebra $A(G)$ of a locally compact (not necessarily abelian) group $G$ (see $[\mathrm{E}]$ ), which is one of the important Banach algebras of harmonic analysis. Our approach and results are quite different from those of the above papers.

Now we summarize the main results of the paper. Let $A$ be a commutative semisimple Banach algebra, $M(A)$ be its multiplier algebra and $T$ be an element of $M(A)$. The Gelfand spectrum $\Delta(M(A))$ of $M(A)$ is the union of the Gelfand spectrum $\Delta(A)$ of $A$ and the hull of $A, A$ being considered as an ideal in $M(A)$ (see [La1]). Denote by $\widehat{T}: \Delta(M(A)) \rightarrow C$ the Gelfand transform of $T$. Let, as above,

$$
\Delta(\widehat{T})=\{f \in \Delta(A): \widehat{T}(f) \neq 0\} \quad \text { and } \quad \delta(T)=\inf \{|\widehat{T}(f)|: f \in \Delta(\widehat{T})\} .
$$

In Section 2 we study the question of when $\delta(T)>0$. We give three characterizations of this condition. The first of these says that if $\Delta(A)$ is closed in $\left(A^{*}, \Upsilon\right)$, then $\delta(T)>0$ iff the set $T^{*}(\Delta(\widehat{T}))$ is closed in $\left(A^{*}, \Upsilon\right)$. Here $\Upsilon$ is any locally convex topology on $A^{*}$ compatible with the duality $\left(A^{*}, A^{* *}\right)$. At this point we recall that if the algebra $A$ has a BAI then $\Delta(A)$ is closed in $\left(A^{*}\right.$, weak) and this may also happen even if $A$ has no (bounded or not) approximate identity (see [ய̈]). The second characterization says that, if the ideal $B=T(A)$ is closed in $A$ and $\Delta(A)$ is closed in $\left(A^{*}\right.$, weak), then $\delta(T)>0$ iff the spectrum of the algebra $B$ is closed in $\left(B^{*}\right.$, weak $)$. At this point we remark that, even if $A$ is unital, the closedness of the ideal $T(A)$ in $A$ does not imply that $\delta(T)>0$. A rather simple example is this. Let $A(D)$ be the classical disk algebra. Then the ideal $A(D) z=\{a \in A(D): a(z)=0\}$ is closed in $A(D)$ but $\delta(z)=0$. The third characterization applies to regular Banach algebras and says this: If $A$ is regular, $\Delta(A)$ is closed in $\left(A^{*}\right.$, weak) and $T(A)$ is closed in $A$, then $\delta(T)>0$ iff the following condition holds: 
There is a constant $c>0$ such that, for each $f \in \Delta(\widehat{T})$, there exists an element $a \in A$ with $\|a\| \leq c$ such that the support of $\widehat{a}$ is contained in $\Delta(\widehat{T})$ and $\langle a, f\rangle=1$.

This condition is satisfied, for instance, for every open subset $E$ of the spectrum of the Fourier algebra $A(G)$. It is also satisfied for every open subset of the spectra of algebras called "boundedly regular" in [L-N; p. 418]. We give an example of a regular Banach algebra $B$ such that the above condition fails for every infinite subset of $\Delta(B)$. In this section we also show that if $A$ is regular, $\Delta(A)$ is closed in $\left(A^{*}\right.$, weak) and there exists a one-to-one weakly compact homomorphism from $A$ into a Banach algebra $B$, then the analog of the Host-Parreau Theorem holds for $A$. Thus the problem of when a multiplier with closed range on a Banach algebra with a BAI factors as the product of an invertible and an idempotent multiplier has its full meaning (and difficulty) only for those algebras $A$ on which there exists no one-to-one weakly compact homomorphism.

In Section 3, we study the following two questions: (a) When is the ideal $T(A)$ closed in $A$ and does it have a BAI? (b) How to characterize the idempotent elements of $M(A)$ in terms of subsets of $\Delta(A)$ ? Concerning the first question, we first give some general results such as this: If $A$ has a BAI, then $T(A)$ is closed in $A$ and has a BAI iff the ideal $T \circ M(A)$ is closed in $M(A)$ and has a unit element. Then we show that when $A$ is a BSE-algebra [T-Ha] with a BAI (the definition of which is recalled below), then $T(A)$ is closed in $A$ and has a BAI iff $T$ is spectrally invertible in the sense that there exists a multiplier $S \in M(A)$ such that $\widehat{T}(f) \widehat{S}(f)=1$ for each $f \in \Delta(\widehat{T})$. As a corollary, we show that if the closed linear span of $\Delta(\widehat{T})$ in $A^{*}$ is isomorphic to $\ell^{1}(\Delta(\widehat{T}))$ then $\delta(T)>0$ iff $T(A)$ is closed in $A$ and has a BAI. One of the main results of this section says that if $A$ is regular, has a BAI and $T(A)$ is closed in $A$ then $T(A)$ has a BAI iff $\Delta(\widehat{T})$ is a $\mathrm{U}$ - (or Urysohn) set with respect to $A$ in the following sense:

There exists a constant $c>0$ such that, for each compact $K \subseteq O$, there exists an $a \in A$ with $\|a\| \leq c$ such that the support of $\widehat{a}$ is contained in $O$ and $\widehat{a}=1$ on $K$.

This notion, which seems to be new in this context, has turned out to be quite important. Indeed, the closed-open U-subsets of $\Delta(A)$ also characterize the idempotent elements of $M(A)$. The following result, which is one of the main results of this paper, can be considered as an abstract analog of Cohen's (and Host's [H]) Idempotent Theorem [C]: Suppose that $A$ is a regular BSE-algebra with a BAI and satisfying Ditkin's condition for synthesis. Then a closed-open subset $E$ of $\Delta(A)$ supports an idempotent 
element of $M(A)$ iff $E$ is a U-set. Thus, for instance, when $A$ is the Fourier algebra of an amenable locally compact group $G$, a closed-open subset of $G$ is a U-set with respect to the Fourier algebra $A(G)$ iff it is in the coset ring of $G$ generated by the open subgroups of $G$.

1. Notation and preliminary results. Our notation and terminology are standard and some of them are already introduced in the preceding section. For any Banach space $X$, we denote by $X^{*}$ the dual space of $X$ and we always consider $X$ as naturally embedded into $X^{* *}$. For $x \in X$ and $f \in X^{*}$, we denote by $\langle x, f\rangle$ (and also $\langle f, x\rangle$ ) the natural duality between $X$ and $X^{*}$. Finally, $L(X)$ denotes the algebra of bounded linear operators on $X$.

Now let $A$ be a commutative semisimple Banach algebra. On $A^{* *}$ there exist two natural multiplications extending that of $A$, known as the first and second Arens products of $A^{* *}$. The space $A^{* *}$ will always be considered as equipped with the first of these products whose definition and main properties are recalled below. For $a, b$ in $A, f$ in $A^{*}$ and $n, m$ in $A^{* *}$, the elements $f a$ and $n f$ of $A^{*}$ and $m n$ of $A^{* *}$ are defined, respectively, as follows:

$$
\langle f a, b\rangle=\langle f, a b\rangle, \quad\langle n f, a\rangle=\langle n, f a\rangle, \quad\langle m n, f\rangle=\langle m, n f\rangle .
$$

Equipped with this multiplication, $A^{* *}$ is a Banach algebra and $A$ is a subalgebra of it. In general $A^{* *}$ is not commutative but, for $a \in A$ and $m \in A^{* *}$, $a m=m a$. This multiplication is in general not separately weak-star to weak-star continuous on $A^{* *}$ but, for $n \in A$ fixed, the mapping $m \mapsto m n$ is continuous in the weak-star topology of $A^{* *}$. If $\left(e_{\alpha}\right)_{\alpha \in I}$ is a BAI (= bounded approximate identity) in $A$ then each weak-star cluster point $u$ of this net in $A^{* *}$ is a right unit in $A^{* *}$ so that, for each $m \in A^{* *}, m u=m$. Conversely, every right unit of $A^{* *}$ is a cluster point in $\left(A^{* *}\right.$, weak-star) of some BAI of $A$. Also, for $m, n \in A^{* *}$ and $f \in \Delta(A),\langle m n, f\rangle=\langle n, f\rangle\langle m, f\rangle$. For all these results we refer the reader to [B-D; Section 28]. For a set $E \subseteq \Delta(A)$, the ideal $\operatorname{ker}(E)$ is defined as $\operatorname{ker}(E)=\bigcap_{f \in E} f^{-1}(0)$; for an ideal $I$ of $A$, the hull of $I$ is defined by hull $(I)=\left\{f \in \Delta(A): I \subseteq f^{-1}(0)\right\}$ (see [La2]).

A (bounded) linear operator $T: A \rightarrow A$ is said to be a multiplier on $A$ if, for each $a, b \in A$, we have $T(a b)=a T(b)$. The space of multipliers on $A$ is denoted by $M(A)$. The space $M(A)$ is a commutative, semisimple unital subalgebra of the algebra $L(A)$ of bounded linear operators on $A$. Each $a \in A$ defines a multiplier $L_{a}: A \rightarrow A$ on $A$ by $L_{a}(x)=a x$. The algebra $A$ being semisimple, identifying $A$ with the ideal $\left\{L_{a}: a \in A\right\}$, we can consider $A$ as an ideal of $M(A)$. However $A$ is in general not closed in $M(A)$. By $\operatorname{Hull}(A)$ we denote the hull of $A$, considered as an ideal in $M(A)$. 
The Gelfand spectrum of $M(A)$ is the set $\Delta(M(A))=\Delta(A) \cup \operatorname{Hull}(A)$ (see [La1]). The set $\Delta(A)$ is open in $\Delta(M(A))$ but in general far from being dense in it. For $T \in M(A)$, we denote by $\widehat{T}: \Delta(M(A)) \rightarrow \mathbb{C}$ the Gelfand transform of $T$. We shall work with the restriction of $\widehat{T}$ to $\Delta(A)$.

For $T \in M(A)$, let $T^{* *}: A^{* *} \rightarrow A^{* *}$ be its second adjoint and $\varphi_{T}$ be the restriction of $\widehat{T}$ to $\Delta(A)$. Then, for $f \in \Delta(A), \varphi_{T}(f)=\widehat{T}(f)$ and $T^{*}(f)=\widehat{T}(f) f$. The second adjoint $T^{* *}$ of $T$ is also a multiplier on $A^{* *}$ $\left(T^{* *}(n m)=n T^{* *}(m)\right)$, and if $u$ is a right unit in $A^{* *}$, for $f \in \Delta(A)$ we have $\varphi_{T}(f)=\left\langle T^{* *}(u), f\right\rangle$. In particular, the function $f \mapsto\left\langle T^{* *}(u), f\right\rangle$ is continuous on the Gelfand spectrum of $A$. Moreover the element $e=T^{* *}(u)$ is such that, for $a \in A$, ae $=a T^{* *}(u)=T^{* *}(a u)=T^{* *}(a)=T(a)$ so that $T=L_{e}$, where $L_{e}: A \rightarrow A$ is the multiplier defined by $L_{e}(a)=a e$. The multiplier $L_{e}$ does not depend on the right unit chosen and $\varphi_{L_{e}}(f)=\langle e, f\rangle$ for $f \in \Delta(A)$. Whenever convenient, we shall identify $T$ and $L_{e}$. This will permit us to use the weak-star topology of $A^{* *}$.

Finally, for a locally compact topological group $G$, we denote by $A(G)$ its Fourier algebra and by $B(G)$ its Fourier-Stieltjes algebra. For the definitions and properties of these algebras, we refer the reader to the paper of P. Eymard [E]. Here we mention some of the well known properties of these algebras that we shall need. The algebra $A(G)$ is a commutative semisimple, regular Tauberian Banach algebra of functions on $G$, with $A(G) \subseteq C_{0}(G)$, the space of continuous functions on $G$ that vanish at infinity. The spectrum of $A(G)$, via the Dirac measures, is $G$. When $G$ is amenable, the algebra $A(G)$ has a BAI and its multiplier algebra is $B(G)$. In any case, $A(G)$ is a closed ideal of $B(G)$, and $A(G)=B(G)$ when $G$ is compact. If $G$ is abelian, then $A(G)$ is isometrically isomorphic to the group algebra $L^{1}(\widehat{G})$, and $B(G)$ to $M(\widehat{G})$.

2. An analysis of the condition $\delta(T)>0$. Throughout the paper $A$ will be a commutative semisimple Banach algebra and $T$ will be a multiplier on $A$. Other hypotheses on $A$ will be made as needed. Let $\Delta(\widehat{T})=\{f \in$ $\Delta(A): \widehat{T}(f) \neq 0\}, \Delta_{0}(\widehat{T})=\{f \in \Delta(A): \widehat{T}(f)=0\}$ and $\delta(T)=\inf \{|\widehat{T}(f)|:$ $f \in \Delta(\widehat{T})\}$. The set $\Delta_{0}(\widehat{T})$ is the hull of the ideal $T(A)$, and when this ideal is closed in $A$, the set $\Delta(\widehat{T})$ is its Gelfand spectrum. The quantity $\delta(T)$ need not be strictly positive; in this section our aim is to find out when $\delta(T)>0$. This section, being long, will be divided into two subsections. We shall go from general to particular. Unless otherwise specified, the space $\Delta(A)$ is equipped with its Gelfand topology.

2.a. The general case. In the first characterization of the condition $\delta(T)>0$ we do not assume that $T(A)$ is closed in $A$. This result shows 
that in this kind of questions, besides weak-star and hull-kernel topologies on $\Delta(A)$ or $\Delta(M(A))$, the weak topology $\sigma\left(A^{*}, A^{* *}\right)$ and the norm topology of $A^{*}$ are also relevant.

We start with a very general result. Let $\Upsilon$ be a locally convex Hausdorff topology on $A^{*}$ for which $T^{*}$ is continuous from $\left(A^{*}, \Upsilon\right)$ to $\left(A^{*}, \Upsilon\right)$. For instance, $\Upsilon$ may be the norm topology, weak topology, weak-star topology or any locally convex topology compatible with $\left(A^{*}, A^{* *}\right)$ or even with $\left(A^{*}, A\right)$. The condition $\delta(T)>0$ is closely related to the closedness of $\Delta(A)$ in $\left(A^{*}, \Upsilon\right)$.

Lemma 2.1. Suppose that $\Delta(A)$ is closed in $\left(A^{*}, \Upsilon\right)$. Then $\delta(T)>0$ iff the set $T^{*}(\Delta(\widehat{T}))$ is closed in $\left(A^{*}, \Upsilon\right)$.

Proof. We first observe that for $f \in \Delta(A), T^{*}(f)=\widehat{T}(f) f$. Then we observe that if a net $\left(\widehat{T}\left(f_{\alpha}\right) f_{\alpha}\right)_{\alpha \in I}$ in $T^{*}(\Delta(A))$ converges in $\left(A^{*}, \Upsilon\right)$ to a functional $g \in A^{*}$ then either $g=0$ or $g$ is of the form $g=\widehat{T}(f) f$ for some $f \in \Delta(A)$. Indeed, if $g \neq 0$, the net $\left(\widehat{T}\left(f_{\alpha}\right)\right)_{\alpha \in I}$ has no subnet that converges to zero. So, being bounded, it has a subnet $\left(\widehat{T}\left(f_{\beta}\right)\right)_{\beta \in J}$ that converges to a complex number $\lambda \neq 0$. This implies that the subnet $\left(f_{\beta}\right)_{\beta \in J}$ converges in $\left(A^{*}, \Upsilon\right)$ to $g / \lambda$. Since, by hypothesis, $\Delta(A)$ is closed in $\left(A^{*}, \Upsilon\right), g / \lambda$ is in $\Delta(A)$. On the other hand, $T^{*}$ being continuous in the topology $\Upsilon$, the net $\left(T^{*}\left(f_{\beta}\right)\right)_{\beta \in J}$ converges in $\left(A^{*}, \Upsilon\right)$ to $T^{*}(g / \lambda)=\widehat{T}(g / \lambda) g / \lambda$, so that $g=\widehat{T}(g / \lambda) g / \lambda$ is of the form $\widehat{T}(f) f$, with $f=g / \lambda$.

From this we conclude that the set $T^{*}(\Delta(\widehat{T}))$ is closed in $\left(A^{*}, \Upsilon\right)$ iff the zero functional is not in the $\Upsilon$-closure of this set. This being observed, it is now clear that if $\delta(T)=0$ then there exist nets in $T^{*}(\Delta(\widehat{T}))$ that converge in $\left(A^{*}, \Upsilon\right)$ to zero so that zero is in the $\Upsilon$-closure of the set $T^{*}(\Delta(\widehat{T}))$, and this set is not closed in $\left(A^{*}, \Upsilon\right)$.

Conversely, if $\delta(T)>0$, then, $\Delta(A)$ being closed in $\left(A^{*}, \Upsilon\right)$, no net in $T^{*}(\Delta(\widehat{T}))$ can converge in $\left(A^{*}, \Upsilon\right)$ to zero so that zero of $A^{*}$ is not in the $\Upsilon$-closure of the set $T^{*}(\Delta(\widehat{T}))$; hence this set is closed in $\left(A^{*}, \Upsilon\right)$.

As mentioned in the introduction, if $A$ has a BAI then $\Delta(A)$ is closed in ( $A^{*}$, weak). At this point we recall that, in general, existence of a BAI in $A$

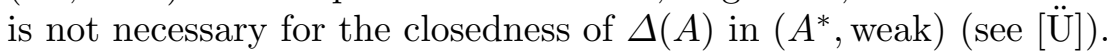

Corollary 2.2. Suppose that $\Delta(A)$ is closed in $\left(A^{*}\right.$, weak). Then $\delta(T)$ $>0$ iff $T^{*}(\Delta(\widehat{T}))$ is closed in $\left(A^{*}\right.$, weak $)$.

Although for lots of interesting Banach algebras, including the Fourier algebra $A(G)$ of any locally compact group $G, \Delta(A)$ is norm closed in $A^{*}$, in general $\Delta(A)$ is not always norm closed in $A^{*}$. Below, in Example 2.13, the reader will find an example of a commutative semisimple regular Banach algebra $B$ whose spectrum is not norm closed in $B^{*}$. In spite of this, 
the assumption that $\Delta(A)$ is norm closed in $A^{*}$ is certainly not a strong hypothesis.

Corollary 2.4. Suppose that $\Delta(A)$ is norm closed in $A^{*}$. Then $\delta(T)$ $>0$ iff the set $T^{*}(\Delta(\widehat{T}))$ is norm closed in $A^{*}$.

Remarks 2.5. (a) Since $\widehat{T}: \Delta(A) \rightarrow \mathbb{C}$ is continuous for the Gelfand topology, when $\delta(T)>0$, the set $\Delta(\widehat{T})$ is both closed and open in $\Delta(A)$. Hence if $\Delta(A)$ is connected, then either $\Delta(\widehat{T})=\Delta(A)$, in which case $T$ is one-to-one on $A$, or $\Delta(\widehat{T})=\emptyset$, in which case $T$ is identically null on $A$. For that reason, the Banach algebras with connected spectrum do not present any interest for us.

(b) Whether $\Delta(A)$ is closed or not in $\left(A^{*}, \Upsilon\right)$, if $T^{*}(\Delta(\widehat{T}))$ is closed in $\left(A^{*}, \Upsilon\right)$ then $\delta(T)>0$. This is clear from the proof of the above lemma.

(c) If $\Delta(\widehat{T})$ is weak-star compact (or compact for $\Upsilon$ ) then $T^{*}(\Delta(\widehat{T}))$ is norm closed in $A^{*}$. Hence, in this case, $\delta(T)>0$. Actually more is true. Indeed, by the Shilov Idempotent Theorem [B-D; Theorem 21.5], there exists an idempotent element $u$ in $A$ which is 1 on the set $\Delta(\widehat{T})$ and zero on the set $\Delta(A) \backslash \Delta(\widehat{T})$. If we put $a=T(u)$ then $T=L_{a}$ so that $T(A)=A a$. Since the spectrum of $L_{a}$ is $\widehat{a}(\Delta(\widehat{T})) \cup\{0\}$ and zero is an isolated point of it, the ideal $T(A)$ is complemented in $A$ and has a unit element [L-M; Corollary 11].

(d) Whenever $\delta(T)>0$, the set $\Delta(\widehat{T})$ is closed in $(\Delta(A)$, weak) if $\Delta(A)$ is closed in $\left(A^{*}\right.$, weak). The converse of this fact is false as the following example shows.

Let $\Omega$ be a locally compact Hausdorff space, $A=C_{0}(\Omega)$ and $a \in A$. Then, since $A$ has a BAI, $\Delta(A)$ is closed in $\left(A^{*}\right.$, weak). Also, since $(\Delta(A)$,

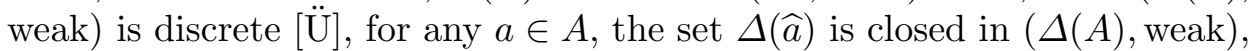
so also in $\left(A^{*}\right.$, weak). However, $\delta(a)=\inf \{|\widehat{a}(f)|: f \in \Delta(\widehat{a})\}>0$ iff $\Delta(\widehat{a})$ is compact. This fact combined with Remark (d) above shows that the closedness of the set $\Delta(\widehat{T})$ in $\left(A^{*}\right.$, weak) is not sufficient, even if $A$ has a BAI, to imply that $\delta(T)>0$.

Now let $G$ be any locally compact group, $a \in B(G) \cap C_{0}(G)$ and $T$ : $A(G) \rightarrow A(G)$ be the multiplier defined by $T(x)=a x$. Then $\delta(T)>0$ iff the set $\Delta(\widehat{a})=\{g \in G: a(g) \neq 0\}$ is compact. Hence, from Remark (c) above, we get the following corollary.

Corollary 2.6. Let $a \in B(G) \cap C_{0}(G)$. Then the ideal $A(G) a$ is complemented in $A(G)$ and unital iff the set $\Delta(\widehat{a})=\{g \in G: a(g) \neq 0\}$ is compact.

As mentioned in the introduction, the spectrum of the algebra $A=A(G)$ is closed in $\left(A^{*}\right.$, weak) iff the group $G$ is amenable [C-X]. On the other hand, 
if the range of a multiplier $S$ on $A$ is dense in $A$ then $\Delta(\widehat{S})=\Delta(A)$. These results imply the following corollary.

COROLlary 2.7. The group $G$ is amenable iff there exists a multiplier $S: A(G) \rightarrow A(G)$ with dense range such that $S^{*}(\Delta(\widehat{S}))$ is closed in $(\operatorname{VN}(G)$, weak).

In the second result characterizing the condition $\delta(T)>0$, we suppose that the ideal $T(A)$ is closed in $A$ and consider $T(A)$ as a Banach algebra in its own right. Recall that, as seen above, closedness of the set $\Delta(\widehat{T})$ in ( $A^{*}$, weak) does not imply that $\delta(T)>0$.

Theorem 2.8. Suppose that $\Delta(A)$ is closed in $\left(A^{*}\right.$, weak) and the ideal $B=T(A)$ is closed in $A$. Then $\delta(T)>0$ iff the spectrum of the algebra $B$ is closed in $\left(B^{*}\right.$, weak $)$.

Proof. First we remark that $\Delta(B)=\Delta(\widehat{T})$. Now suppose that $\delta(T)>0$. Then, by Lemma 2.1 , the set $T^{*}(\Delta(\widehat{T}))$ is weakly closed in $A^{*}$. In particular, zero is not in the weak closure of this set. Hence no net $\left(T^{*}\left(f_{\alpha}\right)\right)_{\alpha \in I}$ in $T^{*}(\Delta(\widehat{T}))$ can converge weakly to zero in $A^{*}$. Since $B^{* *}=T^{* *}\left(A^{* *}\right)$, this implies that no net $\left(f_{\alpha}\right)_{\alpha \in I}$ in $\Delta(\widehat{T})$ can converge weakly in $B^{*}$ to zero. So zero is not in the $\sigma\left(B^{*}, B^{* *}\right)$-closure of $\Delta(B)$. Hence $\Delta(B)$ is closed in $\left(B^{*}\right.$, weak).

Conversely, suppose that $\Delta(B)$ is closed in ( $B^{*}$, weak). Then, since zero is not in $\Delta(B)$, there exists a $\sigma\left(B^{*}, B^{* *}\right)$ neighborhood

$$
V^{m_{1}, \ldots, m_{k}}=\left\{g \in B^{*}:\left|\left\langle m_{i}, g\right\rangle\right|<\varepsilon \text { for } i=1, \ldots, k\right\}
$$

of zero that does not meet $\Delta(B)$. Here $\varepsilon>0$ and $m_{i} \in B^{* *}$. Hence, for each $f \in \Delta(B)$, for at least one $i,\left|\left\langle m_{i}, f\right\rangle\right| \geq \varepsilon$. For each $i=1, \ldots, k$, let

$$
\Delta_{i}=\left\{f \in \Delta(B):\left|\left\langle m_{i}, f\right\rangle\right| \geq \varepsilon\right\} .
$$

Then $\Delta_{1} \cup \ldots \cup \Delta_{k}=\Delta(B)$. Some of $\Delta_{i}$ 's may be empty; dropping them, we can assume that none of $\Delta_{i}$ 's is empty. Since $m_{i} \in B^{* *}$ and $B^{* *}=$ $T^{* *}\left(A^{* *}\right)$, each $m_{i}$ is of the form $m_{i}=T^{* *}\left(n_{i}\right)$ for some $n_{i} \in A^{* *}$. As, for $f \in \Delta_{i}$,

$$
\left|\left\langle T^{* *}\left(n_{i}\right), f\right\rangle\right|=\left|\left\langle n_{i}, T^{*}(f)\right\rangle\right|=\left|\left\langle n_{i}, f\right\rangle\right| \cdot|\widehat{T}(f)| \geq \varepsilon,
$$

we see that the infimum of $|\widehat{T}(f)|$ on $\Delta_{i}$ is strictly positive. Hence, since $\Delta_{1} \cup \ldots \cup \Delta_{k}=\Delta(\widehat{T})$, we conclude that $\delta(T)>0$.

The above result raises naturally the question of exactly when the spectrum of a commutative semisimple Banach algebra $B$ is weakly closed in its dual. If for some $m \in B^{* *}, \inf \{|\langle m, f\rangle|: f \in \Delta(B)\}>0$ then this is the case $[\ddot{\mathrm{U}}]$. For a large class of Banach algebras, the converse is also true. Recall that a commutative semisimple Banach algebra $B$ is said to be self-adjoint 
if, for each $a \in B$, there exists an $a^{*} \in B$ such that $\left\|a^{*}\right\| \leq\|a\|$ and, for $f \in \Delta(B),\left\langle a^{*}, f\right\rangle=\overline{\langle a, f\rangle}$, the complex conjugate of $\langle a, f\rangle$.

Proposition 2.9. Let $B$ be commutative semisimple self-adjoint $B a$ nach algebra. Then $\Delta(B)$ is closed in $\left(B^{*}\right.$, weak) iff there exists an element $m \in B^{* *}$ such that $\inf \{|\langle m, f\rangle|: f \in \Delta(B)\}>0$.

Proof. Suppose that $\Delta(B)$ is closed in $\left(B^{*}\right.$, weak). Then, as the zero functional is not in $\Delta(B)$, for some weak neighborhood $V_{\varepsilon}^{m_{1}, \ldots, m_{k}}$ of zero in $B^{*}$, we have

$$
V_{\varepsilon}^{m_{1}, \ldots, m_{k}} \cap \Delta(B)=\emptyset .
$$

So, for each $f \in \Delta(B),\left|\left\langle m_{i}, f\right\rangle\right| \geq \varepsilon$ for at least one $i=1, \ldots, k$. Let $\left(a_{i, \alpha}\right)_{\alpha \in I}$ be a bounded net in $B$ that converges weak-star in $B^{* *}$ to $m_{i}$. Consider the elements $a_{\alpha}=a_{1, \alpha} a_{1, \alpha}^{*}+\ldots+a_{k, \alpha} a_{k, \alpha}^{*}$ of $B$; the net $\left(a_{\alpha}\right)_{\alpha \in I}$ is bounded. Let $m \in B^{* *}$ be any weak-star cluster point of it in $B^{* *}$. Then,

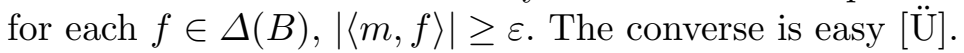

Suppose $A$ has a BAI and $T(A)$ is closed in $A$. Theorem 2.8 shows that the condition $\delta(T)>0$ is necessary for the existence of a BAI in $T(A)$; finding out the conditions under which it is sufficient is the main open problem around which this paper is centered.

2.b. The case where $A$ is regular. The characterizations of the condition $\delta(T)>0$ given above are in a sense "external". In this subsection we give an "internal" characterization; that is, a characterization that does not involve the dual space $A^{*}$. Glicksberg's paper [G] (see also [A-L] and [L-N; p. 418]) also contains a sufficient condition of this type; we shall consider this condition below. In the following definition, the letter $\mathrm{u}$ (both lower case and capital) stands for Urysohn. U-sets will be used in the next section.

Definition 2.10. An open subset $O$ of $\Delta(A)$ is said to be a $u$-set (resp. $U$-set) with respect to $A$ if there exists a constant $c>0$ such that, for each $f \in O$ (resp. for each compact $K \subseteq O$ ), there exists an element $a \in A$ for which $\|a\| \leq c$, the support of $\widehat{a}$ is contained in $O$ and $\widehat{a}(f)=1(\widehat{a}=1$ on $K)$.

Remarks and Examples 2.11. (a) If the algebra $A$ is regular and $O$ is an open subset of $\Delta(A)$, then for each $f \in O$, there is an element $a \in A$ such that the support of $\widehat{a}$ is contained in $O$ and $\widehat{a}(f)=1$ but in general we do not have any control of the norm of $a$. In this definition, the important point is that $\|a\| \leq c$, where the constant $c$, which depends on the open set $O$, does not depend on $f$ (or the compact set $K$ ) in $O$.

(b) If $A=C_{0}(\Omega)$, where $\Omega$ is a locally compact space, then by the Urysohn Lemma, every open subset of $\Omega$ is a U-set (so u-set) with respect to $A$. 
(c) By the Shilov Idempotent Theorem, each compact open subset of $\Delta(A)$ is a $\mathrm{U}$-set with respect to $A$.

(d) Let $G$ be any locally compact group and $A=A(G)$ be its Fourier algebra. Then every open subset $O$ of $G$ is a u-set with respect to $A(G)$. Indeed, let $O$ be an open subset of $G$ and $f$ be an element of $O$. Let $U$ be a compact neighborhood of the identity element of $G$ such that $f U U^{-1} \cap F=\emptyset$, where $F$ is the complement of $O$ in $G$. Let $b=\chi_{U} / \mu(U)$ and $c=\chi_{f U}$. Here $\chi_{U}$ is the characteristic function of $U$ and $\mu$ is the left Haar measure of $G$. The functions $b$ and $c$ are both in $L^{2}(G)$ so that $a=c * b^{\vee}$ (here $b^{\vee}(g)=b\left(g^{-1}\right)$ ) is in $A(G)$, its norm is one, $a(f)=1$ and the support of $a$ is contained in $O$.

(e) At the end of his paper [G] (see also [L-N; p. 418]), Glicksberg considers the following condition.

(I) There is a constant $c>0$ such that, for each $f \in \Delta(A)$ and each neighborhood $O$ of $f$, there is an $a \in A$ for which $\|a\| \leq c$, the support of $\widehat{a}$ is contained in $O$ and $\widehat{a}(f)=1$.

A Banach algebra satisfying this condition is called in [L-N; p. 418] "boundedly regular". This condition involves the whole of the space $\Delta(A)$; whereas being a u-set is a local property, it just concerns the subset $O$ of $\Delta(A)$. Clearly, if $A$ satisfies condition (I), then every open subset of $\Delta(A)$ is a u-set with respect to $A$. As we shall see below, (I) is a strong condition; it implies that the set $\Delta(A)$ under the weak topology is discrete.

(f) If the algebra $A$ has a BAI, or more generally if we have an $m \in A^{* *}$ for which $\inf \{|\langle m, f\rangle|: f \in \Delta(A)\}>0$, then $\Delta(A)$ is a u-set with respect to $A$. Indeed, let $\left(a_{a}\right)_{\alpha \in I}$ be a bounded net in $A$ that converges in the weakstar topology of $A^{* *}$ to $m$. We can suppose that, for each $\alpha \in I,\left\|a_{\alpha}\right\| \leq\|m\|$. Fix an $f \in \Delta(A)$ and choose an $\alpha$ such that $\left|\left\langle a_{\alpha}, f\right\rangle\right| \geq \frac{1}{2}|\langle m, f\rangle|$. Let $a=a_{\alpha} /\left\langle a_{\alpha}, f\right\rangle$. Then $\langle a, f\rangle=1$ and $\|a\| \leq 2\|m\|$.

This last remark shows that a necessary condition for the existence of a BAI in a closed ideal $B$ of $A$ is that $\Delta(B)$ should be a u-set with respect to $B$. However, as part (d) of the above remark shows, this condition is too weak to be sufficient for the existence of a BAI in $B$.

TheOREM 2.12. Suppose that the algebra $A$ is regular, $\Delta(A)$ is weakly closed in $A^{*}$ and the ideal $T(A)$ is closed in $A$. Then $\delta(T)>0$ iff the open set $\Delta(\widehat{T})$ is a u-set with respect to $A$.

Proof. Suppose that $\delta(T)>0$, and let $B=T(A)$. Since, by Theorem 2.8, $\Delta(B)$ is closed in $\left(B^{*}\right.$, weak), there exists a weak neighborhood (with $\varepsilon=1$ and $\left.n_{i}=T^{* *}\left(m_{i}\right) \in B^{* *}\right)$

$$
V^{n_{1}, \ldots, n_{k}}=\left\{g \in B^{*}:\left|\left\langle T^{* *}\left(m_{i}\right), g\right\rangle\right|<1 \text { for } i=1, \ldots, k\right\}
$$


of the zero of $B^{*}$ which does not meet $\Delta(\widehat{T})$. Let $c=\max \left\{\left\|T^{* *}\left(m_{i}\right)\right\|: i=\right.$ $1, \ldots, k\}$, and fix an $f \in \Delta(\widehat{T})$. Then for at least one $i,\left|\left\langle T^{* *}\left(m_{i}\right), f\right\rangle\right| \geq 1$. Choose such an $i$, and let $\left(a_{\alpha}\right)_{\alpha \in I}$ be a net in $B$, bounded by $c$, that converges in $\left(B^{* *}\right.$, weak $\left.^{*}\right)$ to $T^{* *}\left(m_{i}\right)$. Fix an $\alpha$ such that $\left|\left\langle a_{\alpha}, f\right\rangle\right| \geq 1 / 2$. Let $a=$ $a_{\alpha} /\left\langle a_{\alpha}, f\right\rangle$. Then $\|a\| \leq 2 c$ and $\langle a, f\rangle=1$. The support of $\widehat{a}$ is contained in $\Delta(\widehat{T})$ since $a \in T(A)$ and, $\delta(T)$ being strictly positive, $\Delta(\widehat{T})$ is closed in $\Delta(A)$. Hence $\Delta(\widehat{T})$ is a u-set with respect to the algebra $A$.

Conversely, suppose that $\Delta(\widehat{T})$ is a u-set with respect to $A$. So there is a constant $c_{1}>0$ such that, for each $f \in \Delta(\widehat{T})$, there is an $a \in A$ for which $\|a\| \leq c_{1},\langle a, f\rangle=1$ and $\operatorname{supp}(\widehat{a}) \subseteq \Delta(\widehat{T})$. Fix an $f \in \Delta(\widehat{T})$ and let $a \in A$ be as above. Let $I$ be the closure of the ideal

$$
\left\{x \in A: \widehat{x} \text { is null on an open set } O_{x} \text { containing } \Delta_{0}(\widehat{T})\right\} .
$$

Then $I \subseteq T(A)$ since the hull of the closed ideal $T(A)$ is $\Delta_{0}(\widehat{T})$ (see [La2; p. 182, Theorem 8.1.1]). As the support of $\widehat{a}$ is contained in $\Delta(\widehat{T}), \widehat{a}$ is null on an open set $O_{a}$ containing $\Delta_{0}(\widehat{T})$ so that $a \in T(A)$. Hence $a=T(b)$ for some $b \in A$. Since $T(A)$ is closed in $A$, by the Open Mapping Theorem, we can assume that the norm of $b$ is less than a certain constant $c_{2}$, which depends on $c_{1}$ but not on $a$ or $f$. This constant being independent of the $f$ chosen in $\Delta(\widehat{T})$,

$$
1=\langle a, f\rangle=\langle T(b), f\rangle=\widehat{T}(f)\langle b, f\rangle \leq|\widehat{T}(f)| c_{2}
$$

so that $\inf \{|\widehat{T}(f)|: f \in \Delta(\widehat{T})\} \geq 1 / c_{2}$. Hence $\delta(T)>0$.

The example indicated in the introduction (the disk algebra) shows that without regularity the preceding theorem does not hold even if the algebra is unital. The next example shows that, even if $A$ is regular, one direction of this theorem fails if $\Delta(A)$ is not closed in $\left(A^{*}\right.$, weak).

ExAmple 2.13. Let $B$ be the algebra of complex sequences $\varphi: \mathbb{N} \rightarrow \mathbb{C}$ such that $\lim _{n \rightarrow \infty} n \varphi(n)=0$. The space $B$, with the coordinatewise multiplication and the norm $\|\varphi\|=\sup \{n|\varphi(n)|: n \geq 1\}$, is a Banach algebra and $\Delta(B)=\left\{\delta_{n}: n \in \mathbb{N}\right\}$, where $\left\langle\delta_{n}, \varphi\right\rangle=\varphi(n)$. The space $\Delta(B)$ is discrete, so $B$ is regular. If $\left(\varphi_{n}\right)_{n \in \mathbb{N}}$ is any bounded sequence in $B$, say $\left\|\varphi_{n}\right\| \leq c$ for all $n \in \mathbb{N}$, then, since $\left\|\varphi_{n}\right\|=\sup \left\{k\left|\varphi_{n}(k)\right|: k \geq 1\right\} \leq c$, we see that $\left|\varphi_{n}(n)\right| \leq c / n$ for all $n \geq 1$. This shows that no infinite subset of $\Delta(B)$ is a u-set. This also shows that

$$
\left\|\delta_{n}\right\|=\sup \left\{\left|\left\langle\delta_{n}, \varphi\right\rangle\right|:\|\varphi\| \leq 1\right\}=\sup \{|\varphi(n)|:\|\varphi\| \leq 1\}=1 / n .
$$

Hence $\Delta(B)$ is not norm closed, so not weakly closed, in $B^{*}$. The multiplier algebra of $B$ is $\ell^{\infty}$ (see [L-N; p. 303]). Now consider the sequence $e=$ $(1,0,1,0,1, \ldots)$, and let $T: B \rightarrow B$ be the mapping defined by $T(\varphi)=e \varphi$ (pointwise multiplication). Then $T$ is an idempotent multiplier on $B$ so that 
$T(B)$ is closed in $B$. For this multiplier, $\Delta(\widehat{T})=\left\{\delta_{n}: n=2 k+1, k \in \mathbb{N}\right\}$ and $\delta(T)=1$ although $\Delta(\widehat{T})$ is not a u-set with respect to $B$. Observe also that although $T^{2}(B)=T(B), T(B)$ does not have a BAI.

Next we present an implication of the Glicksberg condition (I). As we shall see below this result is closely connected with the problems under study in this paper. In this result we do not assume that $A$ is regular.

Theorem 2.14. Suppose that $A$ satisfies Condition (I) and that $\Delta(A)$ is closed in $\left(A^{*}\right.$, weak $)$. Then every weakly compact homomorphism from $A$ into another commutative semisimple Banach algebra $B$ has a finite-dimensional range.

Proof. By Theorem 2.14 of [ $\ddot{\mathrm{U}}$, it is enough to prove that $\Delta(A)$, equipped with the weak topology induced by that of $A^{*}$, is discrete. By condition (I), there exists a constant $c>0$ such that, for each $f \in \Delta(A)$ and for each neighborhood $V$ of $f$, there is an $a \in A$ with $\|a\| \leq c, \operatorname{supp}(\widehat{a}) \subseteq V$ and $\langle a, f\rangle=1$. Fix an $f \in \Delta(A)$, and let $\left(V_{a}\right)_{a \in I}$ be a basic neighborhood system of $f$ in the Gelfand space $\Delta(A)$, ordered by reverse inclusion. For each $\alpha \in I$, let $a_{\alpha}$ be an element of $A$ such that $\left\|a_{\alpha}\right\| \leq c, \operatorname{supp}(\widehat{a}) \subseteq V_{\alpha}$ and $\left\langle a_{\alpha}, f\right\rangle=1$. Let $m_{f}$ be a weak ${ }^{*}$-cluster point of the net $\left(a_{\alpha}\right)_{\alpha \in I}$ in $A^{* *}$. Then $\left\langle m_{f}, f\right\rangle=1$ and $\left\langle m_{f}, g\right\rangle=0$ for each $g \in \Delta(A), g \neq f$. This implies that $(\Delta(A)$, weak $)$ is discrete.

To avoid long paraphrasing, let us say that a Banach algebra $B$ has the Hewitt property if each multiplier $T$ on $B$ with a closed range factors as a product of an invertible and an idempotent multiplier. Host and Parreau's famous result says that the group algebra $L^{1}(G)$ of any locally compact abelian group $G$ has the Hewitt property.

Theorem 2.15. Suppose that $A$ is regular, $\Delta(A)$ is closed in ( $A$, weak) and every open subset of $\Delta(A)$ is a u-set with respect to $A$. If there exists a one-to-one weakly compact homomorphism from A into some Banach algebra $B$ then $A$ has the Hewitt property.

Proof. Let $h: A \rightarrow B$ be a one-to-one weakly compact homomorphism. Replacing $B$ by $\overline{h(A)}$ we can assume that the range of $h$ is dense in $B$ and $B$ is commutative. Let us first see that the condition that $\Delta(A)$ is closed in ( $A$, weak) forces $A$ to be unital.

To this end, we first show that $h^{*}(\Delta(B))$ is closed in $\Delta(A)$. Indeed, let $\left(h^{*}\left(f_{\alpha}\right)\right)_{\alpha \in I}$ be a net that converges weakly in $A^{*}$ to some $g \in \Delta(A)$. So $g \neq 0$. Passing to a subnet, we can assume that $\left(f_{\alpha}\right)_{\alpha \in I}$ converges in the weak-star topology of $B^{*}$ to a functional $f \in B^{*}$. The fact that $g \neq 0$ implies that $f \neq 0$. So $f \in \Delta(B), g=h^{*}(f)$ is in $h^{*}(\Delta(B))$, and $h^{*}(\Delta(B))$ is closed in $(\Delta(A)$, weak $)$. 
Hence, $h$ being weakly compact, $h^{*}(\Delta(B))$ is weakly compact. In particular, $h^{*}(\Delta(B))$ is closed in the Gelfand space $\Delta(A)$. Now using the fact that $A$ is regular and $h$ is one-to-one, we deduce that $h^{*}(\Delta(B))=\Delta(A)$ so that $\Delta(A)$ is also weakly compact, so weak-star compact. Hence $A$, being semisimple, is unital.

Thus every multiplier on $A$ is of the form $L_{a}$ for some $a \in A$. Now suppose that $L_{a}(A)=A a$ is closed in $A$. Then, by Theorem $2.12, \delta\left(L_{a}\right)>0$. This implies that the set $\Delta(\widehat{a})=\{f \in \Delta(A): \widehat{a}(f) \neq 0\}$ is compact, which in turn implies that the ideal $A a$ is unital. Let $u=a e$ be the unit element of $A a$. Then, as one can readily check, $L_{a}=L_{u} L_{v}$, where $v=a+1-u$ and 1 is the unit element of $A$. Clearly $L_{u}$ is an idempotent multiplier and $L_{v}$ is an invertible multiplier since, as one can easily see, $\widehat{v} \neq 0$ on $\Delta(A)$. Hence $A$ has the Hewitt property.

Thus the Hewitt property is of interest only for those commutative regular algebras on which there is no weakly compact one-to-one homomorphism. The Fourier algebra $A(G)$ of any amenable group is such an algebra (see for instance [ய̈; Corollary 2.16]). On the other hand, as proved by E. Granirer [Gr], if $G$ contains the free group $F_{2}$ then there exist weakly compact homomorphisms on $A(G)$ with infinite-dimensional ranges. We include here the following result, which seems to have been unnoticed.

Proposition 2.16. Let $G$ be any locally compact group. Then every compact homomorphism from $A(G)$ into any Banach algebra $B$ has a finitedimensional range.

Proof. Put $A=A(G)$. We first remark that the set $\Delta(A)$ is norm closed in $A^{*}$ and is discrete under the norm topology since it is discrete under the weak topology [ن̈]. Let $I$ be a closed ideal of $A$ and $\varphi: A \rightarrow A / I$ be the quotient homomorphism. Using this homomorphism we show easily that the spectrum of the algebra $A / I$ is also norm closed and norm discrete in its dual space.

Now let $h: A \rightarrow B$ be a compact homomorphism. Replacing $B$ by $\overline{h(B)}$ we can assume that $B$ is commutative and the range of $h$ is dense. Let $\widetilde{h}: A / \operatorname{Ker} h \rightarrow B$ be the induced homomorphism. Then $\widetilde{h}$ is one-to-one and compact. The algebra $A /$ Ker $h$ being regular, as in the proof of the above theorem, we deduce that its spectrum is norm compact, so finite. Hence the hull of the ideal Ker $h$ is finite. Since one-point subsets of $G$ are sets of synthesis [E; p. 229, Corollary 2], the finite set hull(Ker $h$ ) is a set of synthesis (see e.g. Corollary 5.7 of [Ü]). Then $\operatorname{Ker} h=\operatorname{ker}(\operatorname{hull}(\operatorname{Ker} h)$ ). This implies that the algebra $A / \operatorname{Ker} h$ is semisimple. As the spectrum of $A / \operatorname{Ker} h$ is finite, it is finite-dimensional. Hence the dimension of $h(A)$ is also finite. 
3. Existence of BAI in $T(A)$ and idempotent elements of $M(A)$. In this section we present an analysis of the following two problems: (a) When is the ideal $T(A)$ closed in $A$ and does it have a BAI? (b) How to characterize the idempotent elements of $M(A)$ in terms of subsets of $\Delta(A)$ ? We first give a couple of general results and then consider these two problems in more special settings.

3.a. Existence of BAI in $T(A)$. Let $G$ be a locally compact abelian group and $\mu \in M(G)$ be a regular Borel measure on $G$. As proved by Glicksberg [G], the ideal $L^{1}(G) * \mu$ is closed in $L^{1}(G)$ iff the ideal $M(G) * \mu$ is closed in $M(G)$. Moreover, by the Host-Parreau Theorem, in this case the ideal $L^{1}(G) * \mu$ has a BAI. The next proposition shows that this is a general result.

Proposition 3.1. Suppose that $A$ has a BAI. Then the ideal $T(A)$ is closed in $A$ and has a BAI iff the ideal $T \circ M(A)=\{T \circ S: S \in M(A)\}$ is closed in $M(A)$ and has a unit element.

Proof. (a) $\Rightarrow(\mathrm{b})$. Suppose that $T(A)$ is closed in $A$ and has a BAI. Then by the Cohen-Hewitt Factorization Theorem [He-R; 32.22], $A A=A$ and $T(A) T(A)=T(A)$. Hence $T^{2}(A)=T(A)$. This implies that $T$ factors as $T=S \circ \theta=\theta \circ S$ (see [L-M; Theorems 5 and 6]), where the multiplier $S$ is invertible and $\theta$ is idempotent. Hence $T \circ M(A)=\theta \circ M(A)$ is closed in $M(A)$ and $\theta \circ I$ is the unit element of $T \circ M(A)$, where $I$ is the unit element of $M(A)$.

(b) $\Rightarrow($ a). Suppose that $T \circ M(A)=\{T \circ S: S \in M(A)\}$ is closed in $M(A)$ and has a unit element, say $T \circ U$. As $T \circ I=T \circ I \circ T \circ U=$ $T^{2} \circ U$, we have $T(A)=T^{2} \circ U(A) \subseteq T^{2}(A)$. Hence $T(A)=T^{2}(A)$. This implies that $T(A)$ is closed in $A$ and has a BAI [L-M; Theorems 5 and 6$]$.

Theorem 3.2. Suppose that $A$ has a BAI. Then $T(A)$ is closed in $A$ and has a BAI iff there is a multiplier $S \in M(A)$ such that $\widehat{T} \widehat{S}=1$ on $\Delta(\widehat{T})$.

Proof. Suppose first that $T(A)$ is closed and has a BAI. Then as in the preceding proof, $T$ factors as $T=B \circ \theta$, where $B$ is invertible in $M(A)$ and $\theta$ is an idempotent of $M(A)$. Let $S$ be the inverse of $B$. Then, since $\Delta(\widehat{T})=\Delta(\widehat{\theta}), \widehat{T} \widehat{S}=1$ on $\Delta(\widehat{T})$.

Conversely, suppose that there is an $S \in M(A)$ such that $\widehat{T} \widehat{S}=1$ on $\Delta(\widehat{T})$. Put $\theta=T \circ S$. Then $\widehat{\theta}=1$ on $\Delta(\widehat{T})$ and $\widehat{\theta}=0$ on $\Delta_{0}(\widehat{T})$. Hence, $A$ being semisimple, for each $a \in A, \theta(a)=\theta^{2}(a)$ so that $\theta$ is an idempotent multiplier (so a homomorphism), $\theta(A)$ is closed in $A$ and has a BAI. Hence it is enough to show that $T(A)=\theta(A)$. We have $\theta(A)=T(S(A)) \subseteq T(A)$. To prove the reverse inclusion, we first remark that, again by semisimplicity of $A$, for each $a \in A, T(a)=T(\theta(a))$. Hence $T(A)=T(\theta(A))=\theta(T(A)) \subseteq$ 
$\theta(A)$ so that $T(A)=\theta(A)$, and therefore $T(A)$ is closed in $A$ and has a BAI.

In view of this result it is convenient to introduce the following notion.

We say that a multiplier $T \in M(A)$ is spectrally invertible if there is a multiplier $S \in M(A)$ such that $\widehat{T} \widehat{S}=1$ on $\Delta(\widehat{T})$.

In this terminology, the preceding theorem says that a commutative semisimple Banach algebra $B$ with a BAI has the Hewitt property iff every multiplier $S \in M(B)$ with closed range is spectrally invertible. It is also clear that if $S$ is spectrally invertible then $S(B)$ is complemented in $B$ and $\delta(S)>0$. To continue, we need the following notion introduced by Takahasi and Hatori in [T-Ha].

A commutative semisimple Banach algebra $B$ with a BAI is said to be a BSE-algebra (= Bochner-Schoenberg-Eberlein) if any $u \in B^{* *}$ for which $\widehat{u}: \Delta(B) \rightarrow \mathbb{C}$ is continuous on $\Delta(B)$ belongs to the set

$$
\Lambda(B)=\left\{m \in B^{* *}: m B \subseteq B\right\} .
$$

Here $\widehat{u}$ is defined by $\widehat{u}(f)=\langle u, f\rangle$.

As proved in [T-Ha], besides the commutative group algebra $L^{1}(G)$ and the $C^{*}$-algebra $C_{0}(\Omega)$, the classical disk algebra $A(D)$ and Hardy space $H^{\infty}(D)$ are BSE-algebras. For an amenable locally compact group $G$, by Corollary 2 of [E; p. 202], the Fourier algebra $A(G)$ is also a BSE-algebra.

The next result gives a necessary and sufficient condition for $T$ to be spectrally invertible.

TheOrem 3.3. Suppose A is a BSE-algebra with a BAI. Then $T$ is spectrally invertible iff there exists a constant $c>0$ such that

$$
\forall \lambda_{1}, \ldots, \lambda_{n} \in \mathbb{C}, \forall f_{1}, \ldots, f_{n} \in \Delta(\widehat{T}), \quad\left|\sum_{i=1}^{n} \lambda_{i}\right| \leq c\left\|\mid \sum_{i=1}^{n} \lambda_{i} \widehat{T}\left(f_{i}\right) f_{i}\right\| .
$$

Proof. Let $u \in A^{* *}$ be a right unit for the Arens product of this space. This is just a weak-star cluster point in $A^{* *}$ of a BAI of $A$. Put $e=T^{* *}(u)$, and let $L_{e}: A \rightarrow A$ be the multiplier defined by $L_{e}(a)=a e$. Then $T=L_{e}$ and, for $f \in \Delta(A), \widehat{T}(f)=\langle e, f\rangle$. We shall work with $e$ instead of $T$.

Suppose first that $T$ is spectrally invertible. So there exists an $S \in M(A)$ such that $\widehat{T} \widehat{S}=1$ on $\Delta(\widehat{T})$. Let $\widetilde{e} \in A^{* *}$ be the element defined by $\widetilde{e}=$ $S^{* *}(u)$. Then, for each $f \in \Delta(\widehat{T}),\langle\widetilde{e}, f\rangle\langle e, f\rangle=1$. Let complex numbers $\lambda_{1}, \ldots, \lambda_{n}$ and functionals $f_{1}, \ldots, f_{n} \in \Delta(\widehat{T})$ be given. Then

$$
\left\|\sum_{i=1}^{n} \lambda_{i}\left\langle e, f_{i}\right\rangle f_{i}\right\| \geq \frac{1}{\|\widetilde{e}\|}\left|\sum_{i=1}^{n} \lambda_{i}\left\langle e, f_{i}\right\rangle\left\langle f_{i}, \widetilde{e}\right\rangle\right|=\frac{1}{\|\widetilde{e}\|}\left|\sum_{i=1}^{n} \lambda_{i}\right|
$$


so that

$$
\left|\sum_{i=1}^{n} \lambda_{i}\right| \leq\|\widetilde{e}\|\left\|\sum_{i=1}^{n} \lambda_{i}\left\langle e, f_{i}\right\rangle f_{i}\right\| .
$$

Hence $\left|\sum_{i=1}^{n} \lambda_{i}\right| \leq c\left\|\sum_{i=1}^{n} \lambda_{i} \widehat{T}\left(f_{i}\right) f_{i}\right\|$ with $c=\|\widetilde{e}\|$.

Conversely, suppose that

$$
\begin{aligned}
\exists c>0 \forall \lambda_{1}, \ldots, \lambda_{n} & \in \mathbb{C}, \forall f_{1}, \ldots, f_{n} \in \Delta(\widehat{T}), \\
& \left|\sum_{i=1}^{n} \lambda_{i}\right| \leq c|| \sum_{i=1}^{n} \lambda_{i} \widehat{T}\left(f_{i}\right) f_{i} \| .
\end{aligned}
$$

Define a linear functional $m$ on the linear $\operatorname{span} \operatorname{span}(\Delta(\widehat{T}))$ of $\Delta(\widehat{T})$ in $A^{*}$ by

$$
m\left(\sum_{i=1}^{n} \lambda_{i} f_{i}\right)=\sum_{i=1}^{n} \lambda_{i} /\left\langle e, f_{i}\right\rangle
$$

By the above condition, $m$ is bounded. Let $\widetilde{m}$ be a Hahn-Banach extension of $m$ to $A^{*}$ so that $\widetilde{m} \in A^{* *}$. Then, for $f \in \Delta(\widehat{T}),\langle\widetilde{m}, f\rangle\langle e, f\rangle=1$. From this equality and the fact that $\langle e, f\rangle=\left\langle T^{* *} u, f\right\rangle$, we conclude that $\delta(T)>0$, the set $\Delta(\widehat{T})$ is both open and closed in $\Delta(A)$ and the mapping $f \mapsto\langle\widetilde{m}, f\rangle$ is continuous on $\Delta(A)$. Hence, $A$ being a BSE-algebra, $A \widetilde{m} \subseteq A$ and $S=L_{\widetilde{m}}$ is a multiplier on $A$. Since $\widehat{T} \widehat{S}=1$ on $\Delta(\widehat{T}), T$ is spectrally invertible.

As mentioned above, if $T$ is spectrally invertible then $\delta(T)>0$. The next corollary says that if $\Delta(\widehat{T})$ is a "Sidon set" and $\delta(T)>0$ then $T$ is spectrally invertible. At this point we remark that if $A=C_{0}(\Omega)$ and $b$ is a bounded continuous function on $\Omega$ and $T$ is the multiplier defined on $C_{0}(\Omega)$ by $T(x)=b x$ then, as one can easily see, $\overline{\operatorname{span}}(\Delta(\widehat{T}))=\ell^{1}(\Delta(\widehat{T}))$.

Corollary 3.4. Suppose that $A$ is a BSE-algebra with a BAI. Then, if the closed linear span of $\Delta(\widehat{T})$ in $A^{*}$ is isomorphic to the space $\ell^{1}(\Delta(\widehat{T}))$ then $T$ is spectrally invertible if (and only if) $\delta(T)>0$.

Proof. If $\overline{\operatorname{span}}(\Delta(\widehat{T}))$ is isomorphic to $\ell^{1}(\Delta(\widehat{T}))$ then there exists a constant $c>0$ such that

$$
\forall \lambda_{1}, \ldots, \lambda_{n} \in \mathbb{C}, \forall f_{1}, \ldots, f_{n} \in \Delta(\widehat{e}), \quad\left\|\sum_{i=1}^{n} \lambda_{i} f_{i}\right\| \geq c \sum_{i=1}^{n}\left|\lambda_{i}\right| .
$$

So, if $\delta(T)>0$, then

$$
\left\|\sum_{i=1}^{n} \lambda_{i} \widehat{T}\left(f_{i}\right) f_{i}\right\| \geq c \sum_{i=1}^{n}\left|\lambda_{i} \widehat{T}\left(f_{i}\right)\right| \geq c \delta(T) \sum_{i=1}^{n}\left|\lambda_{i}\right| \geq c \delta(T)\left|\sum_{i=1}^{n} \lambda_{i}\right| .
$$

Hence, by the preceding theorem, $T$ is spectrally invertible. 
Next we present a characterization of the spectrally invertible multipliers in terms of U-subsets of $\Delta(A)$. We recall that, as defined in Section 2 above, an open subset $O$ of $\Delta(A)$ is said to be a U-set (or Urysohn set) with respect to $A$ if

$$
\begin{aligned}
& \exists c>0 \forall K \subseteq O \text { compact } \exists a \in A: \\
& \|a\| \leq c, \quad \operatorname{supp}(\widehat{a}) \subseteq O, \quad \widehat{a}=1 \quad \text { on } K .
\end{aligned}
$$

If $B$ is a closed subalgebra of $A$ and the element $a$ above is in $B$ then we say that $O$ is a $U$-set with respect to $B$. It is clear that if an open subset $O$ of $\Delta(A)$ is a U-set with respect to a closed subalgebra $B$ then it is also a U-set with respect to $A$.

We first give a general necessary and sufficient condition for the existence of a BAI in a class of Banach algebras in terms of U-sets. This result is of independent interest.

Lemma 3.5. Let $B$ be a commutative, semisimple, regular Tauberian Banach algebra. Then $B$ has a BAI iff $\Delta(B)$ is a $U$-set with respect to $B$.

Proof. First assume that $B$ has a BAI, bounded by a certain constant $\beta>0$. Let $K$ be a nonempty compact subset of $\Delta(B)$, and $0<\varepsilon<1$ be a fixed number. By regularity of $B$, there is an $a \in B$ such that $\widehat{a}=1$ on $K$. By the Cohen-Hewitt Factorization Theorem [He-R; 32.22], there exist $a_{0}$ and $u$ in $B$ such that $a=a_{0} u$ with $\|u\| \leq \beta$ and $\left\|a-a_{0}\right\| \leq \varepsilon$. Put

$$
b=u+u\left(a-a_{0}\right) .
$$

Then $\widehat{b}=1$ on $K$ and $\|b\| \leq \beta+\varepsilon$. Hence, with $c=\beta+1, \Delta(B)$ is a U-set with respect to $B$. (Note that for the proof of this implication we did not use the fact that $B$ is Tauberian.)

Conversely, suppose that $\Delta(B)$ is a $\mathrm{U}$-set with respect to $B$ so that there exists a constant $c>0$ such that

$$
\forall K \subseteq \Delta(B) \text { compact } \exists a \in A: \quad\|a\| \leq c \quad \text { and } \quad \widehat{a}=1 \quad \text { on } K .
$$

Let $\Sigma$ be the set of compact subsets of $\Delta(B)$. Ordered by inclusion, $\Sigma$ is a directed set. For each $K \in \Sigma$, there exists an $a_{K} \in B$ such that $\left\|a_{K}\right\| \leq c$ and $\widehat{a}_{K}=1$ on $K$. Then the net $\left(a_{K}\right)_{K \in \Sigma}$ is a BAI in $B$. Indeed, let $b$ be an element of $B$ with compact support, and $K_{0}=\operatorname{supp}(\widehat{b})$. Then, for $K \in \Sigma$ with $K \supseteq K_{0}, a_{K} b=b$. As $B$ is Tauberian, from this we conclude that $B$ has a BAI.

This lemma applied to the algebra $A(G)$ shows that a locally compact group $G$ is amenable iff $G$ is a $\mathrm{U}$-set with respect to the algebra $A(G)$. As a first corollary of this lemma we give the following result. 
Corollary 3.6. Suppose that $A$ is regular, and $E \subseteq \Delta(A)$ is a closed set of synthesis. Then the ideal $B=\operatorname{ker}(E)$ has a BAI iff the set $O=$ $\Delta(A) \backslash E$ is a $U$-set with respect to $A$.

Proof. First we remark that the ideal $B=\operatorname{ker}(E)$, regarded as a Banach algebra in its own right, is a commutative semisimple regular Banach algebra. As $E$ is a set of synthesis, $B$ is also Tauberian. If $B$ has a BAI then, by the preceding lemma, $\Delta(B)=\Delta(A) \backslash E$ is a U-set with respect to $B$, so with respect to $A$.

Conversely, suppose that $O$ is a $\mathrm{U}$-set with respect to $A$. Then, since any $a \in A$ for which the support of $\widehat{a}$ is contained in $\Delta(A) \backslash E$ is in the ideal $B$, the set $\Delta(A) \backslash E$ is also a U-set with respect to $B$. Hence, by the preceding lemma, $B$ has a BAI.

The following theorem is one of the main results of this section. We recall that in a commutative semisimple regular Tauberian Banach algebra $B$ with a BAI and satisfying the Dinkin conditions for synthesis [La; p. 206], all closed-open subsets of $\Delta(B)$ are sets of synthesis.

Theorem 3.7. Suppose that $A$ is a regular, has a BAI and all closedopen subsets of $\Delta(A)$ are sets of synthesis. Then the multiplier $T$ is spectrally invertible iff $T(A)$ is closed in $A$ and the set $\Delta(\widehat{T})$ is a U-set with respect to $A$.

Proof. Suppose first that $T$ is spectrally invertible. Then by Theorem 3.2 above, $T(A)$ is closed and has a BAI. Moreover, by Theorem $2.8, \delta(T)>0$ so that the sets $\Delta(\widehat{T})$ and $\Delta_{0}(\widehat{T})$ are both closed and open. Therefore $\Delta_{0}(\widehat{T})$ is a set of synthesis and $T(A)=\operatorname{ker}\left(\Delta_{0}(\widehat{T})\right)$. Since $\Delta_{0}(\widehat{T})$ is a set of synthesis, $T(A)$, as a Banach algebra in its own right, is Tauberian. On the other hand, $T(A)$, being an ideal of $A$, is regular. Hence $B=T(A)$ satisfies the hypothesis of the preceding lemma so that $\Delta(B)=\Delta(\widehat{T})$ is a U-set with respect to $B$, so with respect to $A$.

Conversely, suppose that $\Delta(\widehat{T})$ is a U-set with respect to $A$. Then, by Theorem $2.12, \delta(T)>0$. Hence $\Delta_{0}(T)$ is closed-open in $\Delta(A)$, so a set of synthesis, $T(A)=\operatorname{ker}\left(\Delta_{0}(\widehat{T})\right)$ and $B=T(A)$, viewed as a Banach algebra in its own right, is a commutative, semisimple, regular Tauberian algebra. Now, since $\Delta(\widehat{T})$ is a U-set with respect to $A$, there is a constant $c>0$ such that, for each compact $K$ of $\Delta(\widehat{T})$, there is an $a \in A$ such that

$$
\|a\| \leq c, \quad \operatorname{supp}(\widehat{a}) \subseteq \Delta(\widehat{T}) \quad \text { and } \quad \widehat{a}=1 \quad \text { on } K .
$$

Since $\operatorname{supp}(\widehat{a}) \subseteq \Delta(\widehat{T})$ and $T(A)=\operatorname{ker}\left(\Delta_{0}(\widehat{T})\right)$, we see that $a \in T(A)$. Hence $\Delta(\widehat{T})$ is a U-set with respect to $T(A)$. Hence, by the preceding lemma, $T(A)$ has a BAI and, by Theorem $3.2, T$ is spectrally invertible. 
Since for an amenable locally compact group $G$ the algebra $A=A(G)$ satisfies the Ditkin conditions for synthesis (see for instance [F; Lemma 5.2]), we have the following result.

Corollary 3.8. Let $G$ be an amenable locally compact group, $A=$ $A(G)$ and $u \in B(G)$. Then the following assertions are equivalent.

(a) The ideal $A(G) u$ is closed in $A(G)$ and has a BAI.

(b) $B(G) u$ is closed in $B(G)$ and has a unit element.

(c) $u$ is spectrally invertible, i.e. there exists a $v \in B(G)$ such that $u v=1$ on the set $\Delta(\widehat{u})=\{f \in G: u(f) \neq 0\}$.

(d) The ideal $A(G) u$ is closed in $A(G)$ and $\Delta(\widehat{u})$ is a U-set with respect to $A(G)$.

(e) There is a constant $c>0$ such that, for $\lambda_{1}, \ldots, \lambda_{n} \in \mathbb{C}$ and $f_{1}, \ldots, f_{n}$ $\in \Delta(\widehat{u})$, in the norm of $\operatorname{VN}(G)$, we have

$$
\left|\sum_{i=1}^{n} \lambda_{i}\right| \leq c\left\|\sum_{i=1}^{n} \lambda_{i} u\left(f_{i}\right) f_{i}\right\| .
$$

We also remark that, with the notation of this corollary (and for $G$ amenable), if the closed linear span of $\Delta(\widehat{u})$ in $\mathrm{VN}(G)$ is isomorphic to the space $\ell^{1}(\Delta(\widehat{u}))$ and $\delta(u)>0$ then, by Corollary $3.4, \Delta(\widehat{u})$ is a U-set with respect to $A(G)$ and the ideal $A(G) u$ is complemented in $A(G)$.

3.b. U-sets and idempotent elements of $M(A)$. For a locally compact abelian group $G$, the idempotent elements of $M(G)$ have been characterized by P. Cohen [C]; and in the noncommutative case, those of $B(G)$ by B. Host $[\mathrm{H}]$. The idempotent elements of $M(G)$ (resp. $B(G)$ ) correspond (via Fourier-Stieltjes transform) to the characteristic functions of the elements of the coset ring of $\widehat{G}$ (resp. $G$ ) generated by the cosets of the open subgroups of $\widehat{G}$ (resp. $G$ ). The characterization of the idempotent elements of $M(A)$, for an abstract Banach algebra $A$, presents a certain interest since this is connected with several other problems such as the characterization of the complemented ideals of $A$ and that of the multipliers with closed range.

In this section we give a result which shows that, for a fairly large class of Banach algebras, the idempotent elements of $M(A)$ correspond to the closed-open U-subsets of $\Delta(A)$ in the same way as the idempotent elements of $M(G)$ correspond to the elements of the coset ring of $\widehat{G}$.

As is well known, every open subgroup $H$ of a locally compact group $G$ is also closed; so are the cosets of $H$. The cosets of the open subgroups of $G$ generate the so-called coset ring of $G$; the elements of this ring are both closed and open in $\widehat{G}$. The following can be considered as an abstract analog of this ring. 
Lemma 3.9. Suppose that $A$ is a regular BSE-algebra with a BAI and that closed-open subsets of $\Delta(A)$ are sets of synthesis. Then the set $\Re(\Delta(A))$ of closed-open U-subsets of $A$ forms a ring.

Proof. We first remark that, since $A$ has a BAI, by Lemma $3.5, \Delta(A)$ is a U-set with respect to $A$. Let now $O_{1}$ and $O_{2}$ be two closed-open U-subsets of $\Delta(A)$, and $K$ be a compact subset of $O_{1} \cup O_{2}$. Since $\Delta(A)$ is a locally compact space, we can write $K$ as a union of two compact sets $K_{1}$ and $K_{2}$, with $K_{i} \subseteq O_{i}(i=1,2)$. Since $O_{1}$ and $O_{2}$ are U-sets, there exist constants $c_{i}>0$ and elements $a_{i}(i=1,2)$ in $A$ such that $\left\|a_{i}\right\| \leq c_{i}, \operatorname{supp}\left(\widehat{a_{i}}\right) \subseteq O_{i}$ and $\widehat{a_{i}}=1$ on $K_{i}$. Let $a=a_{1}+a_{2}-a_{1} a_{2}$. Then $\|a\| \leq c_{1}+c_{2}+c_{1} c_{2}$, $\operatorname{supp}(\widehat{a}) \subseteq O_{1} \cup O_{2}$ and $\widehat{a}=1$ on $K$, so that $O_{1} \cup O_{2}$ is a U-set with respect to $A$.

Now let $O$ be a closed-open U-subset of $\Delta(A)$, and $E=\Delta(A) \backslash O$. Then $E$, being closed and open in $\Delta(A)$, is a set of synthesis. Hence the ideal $B=\operatorname{ker}(E)$ of $A$, viewed as a Banach algebra in its own right, is semisimple, regular and Tauberian. Since $\Delta(B)=O$ and since any $a \in A$ with $\operatorname{supp}(\widehat{a}) \subseteq O$ is in $B, \Delta(B)$ is also a U-set with respect to $B$. Hence, by Lemma $3.5, B$ has a BAI. It follows that $B^{* *}$ has a right unit $e$. Clearly $\widehat{e}$ is just the characteristic function of $O$, so it is continuous on $\Delta(A)$. Hence, $A$ being a BSE-algebra, $A e \subseteq A$. As the multiplier defined by $e$ is idempotent, the ideal $A e$ is closed in $A$. Since the hulls of the ideals $A e$ and $B$ are the same, namely $E$, and $E$ is a set of synthesis, $B=A e$. Now let $\left(d_{\alpha}\right)_{\alpha \in I}$ be a BAI for $A$. Put $e_{\alpha}=d_{\alpha}-e d_{\alpha}$. Then the net $\left(e_{\alpha}\right)_{\alpha \in I}$ is in the ideal $I=\operatorname{ker}(O)$ and a BAI for it. Hence, by Lemma 3.5, $E$ is a U-set with respect to $I$, so with respect to $A$. Thus the set $\Re(\Delta(A))$ is a ring.

The next result is the second main result of this section. A subset $O$ of $\Delta(A)$ is said to support an idempotent multiplier if $O=\Delta(\widehat{\theta})$ for some idempotent element $\theta$ of the algebra $M(A)$.

TheOREM 3.10. Suppose that $A$ is a regular BSE-algebra with a BAI satisfying Ditkin's condition for synthesis. Then a closed-open subset $O$ of $\Delta(A)$ supports an idempotent element of $M(A)$ iff $O$ is a $U$-set with respect to $A$.

Proof. Let $\theta \in M(A)$ be an idempotent multiplier and $O=\Delta(\widehat{\theta})$. Then the ideal $\theta(A)$ has a BAI since $A$ has one. Moreover, since $\theta(A)=\operatorname{ker}(E)$, where $E=\Delta(A) \backslash O$, and since $E$ is a set of synthesis, the algebra $B=\theta(A)$ is a regular Tauberian Banach algebra with a BAI. Hence $O$ is a $\mathrm{U}$-set with respect to $B$, so with respect to $A$.

Conversely, let $O$ be a closed-open U-subset of $\Delta(A)$. Then, as we have seen in the proof of the above lemma, the set $E=\Delta(A) \backslash O$ is also a 
U-set with respect to $A$ and, for some $e \in A^{* *}$ for which $A e \subseteq A$, we have $\operatorname{ker}(E)=A e$. Moreover the multiplier defined by $e$ is idempotent and, with $\theta=L_{e}, \Delta(\widehat{\theta})=O$. Thus there is a one-to-one correspondence between the idempotent elements of $M(A)$ and the elements of the ring of closed-open U-subsets of $\Delta(A)$.

Host's Idempotent Theorem combined with the preceding result gives the following corollary.

Corollary 3.11. Let $G$ be an amenable locally compact group and $O$ be a closed-open subset of $G$. Then $O$ is in the coset ring of $G$ iff $O$ is a $U$-set with respect to the algebra $A(G)$.

The preceding corollary and Lemma 3.6 show that the following result holds.

Corollary 3.12. Suppose that $G$ is amenable and $E$ is a closed-open subset of $G$. Then the ideal $I=\operatorname{ker}(E)$ of $A(G)$ has a BAI iff the set $E$ is a U-set with respect to $A(G)$. If this is the case, $I=A(G) u$ for some idempotent $u \in B(G)$.

In particular, if $G$ is discrete then, for a subset $E$ of $G$, the ideal $I=$ $\operatorname{ker}(E)$ has a BAI iff $E$ is in the coset ring of $G$. In this case it is complemented in $A(G)$. We finish with the following question. Let $G$ be an amenable locally compact group and $u \in B(G)$ be such that $A(G) u$ is closed in $A(G)$. Then, by Theorem $2.12, \delta(u)>0$ so that the set $\Delta(\widehat{u})=\{f \in G$ : $u(f) \neq 0\}$ is both closed and open in $G$, so a set of synthesis. One important question that remains unsolved is this. Is $\Delta(\widehat{u})$ a U-set with respect to $A(G)$ ?

\section{References}

[A-L] P. Aiena and B. K. Laursen, Multipliers with closed range on regular commutative Banach algebras, Proc. Amer. Math. Soc. 121 (1994), 1039-1408.

[B-D] F. F. Bonsall and J. Duncan, Complete Normed Algebras, Springer, New York, 1973.

[C-X] C. Chou and G. Xu, The weak closure of the set of left translation operators, Proc. Amer. Math. Soc. 127 (1999), 465-471.

[C] P. Cohen, On a conjecture of Littlewood and idempotent measures, Amer. J. Math. 82 (1960), 191-212.

[E] P. Eymard, L'algèbre de Fourier d'un groupe localement compact, Bull. Soc. Math. France 92 (1964), 181-236.

[F] B. Forrest, Amenability and bounded approximate identities in ideals of $A(G)$, Illinois J. Math. 34 (1990), 1-25.

[G] I. Glicksberg, When is $\mu * L^{1}(G)$ closed?, Trans. Amer. Math. Soc. 72 (1971), 419-425. 
[Gr] E. Granirer, When quotients of the Fourier algebra $A(G)$ are ideals in their duals and when $A(G)$ has WCHP, Math. Japonica 46 (1997), 69-72.

[He-R] E. Hewitt and K. A. Ross, Abstract Harmonic Analysis II, Springer, 1970.

$[\mathrm{H}] \quad$ B. Host, Le théorème des idempotents dans $B(G)$, Bull. Soc. Math. France 114 (1986), 215-223.

[H-P] B. Host et F. Parreau, Sur un problème de I. Glicksberg: Les idéaux fermés de type fini de $M(G)$, Ann. Inst. Fourier (Grenoble) 28 (1978), no. 3, 143164.

[La1] R. Larsen, Theory of Multipliers, Springer, Berlin, 1971.

[La2] -, Banach Algebras, Dekker, New York, 1973.

[L-M] K. B. Laursen and M. Mbekhta, Closed range multipliers and generalized inverses, Studia Math. 107 (1993), 127-135.

[L-N] K. B. Laursen and M. M. Neumann, An introduction to Local Spectral Theory, Clarendon Press, Oxford, 2000.

[T-Ha] S. E. Takahasi and O. Hatori, Commutative Banach algebras which satisfy a Bochner-Schoenberg-Eberlein type theorem, Proc. Amer. Math. Soc. 110 (1990), 149-158.

[ய̈] A. Ülger, Some results about the spectrum of commutative Banach algebras under the weak topology and applications, Monatsh. Math. 121 (1996), 353-379.

[Z] Y. Zaïem, Opérateurs de convolution d'image fermée et unités approchées, Bull. Sci. Math. 99 (1975), 65-74.

Department of Mathematics

Koc University

Fener Yolu

80910, Sariyer, Istanbul, Turkey

E-mail: aulger@ku.edu.tr

Received May 29, 2001

Revised version February 28, 2002 